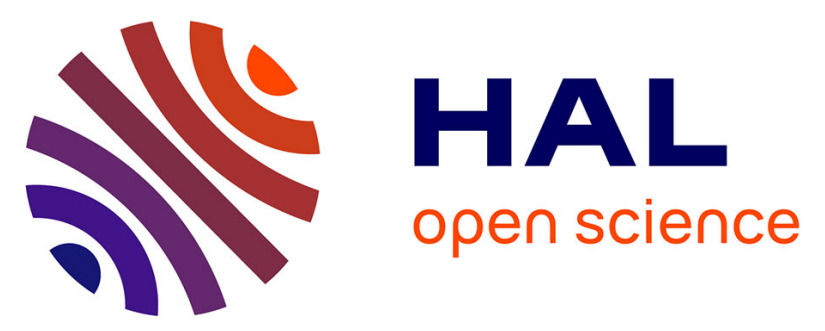

\title{
Anticipation of ETX Metric to manage Mobility in Ad Hoc Wireless Networks
}

Sabrina Naimi, Anthony Busson, Véronique Vèque, Larbi Ben Hadj Slama, Ridha Bouallegue

\section{- To cite this version:}

Sabrina Naimi, Anthony Busson, Véronique Vèque, Larbi Ben Hadj Slama, Ridha Bouallegue. Anticipation of ETX Metric to manage Mobility in Ad Hoc Wireless Networks. Ad Hoc Now, Jun 2014, Benidorm, Spain. pp.29-42, 10.1007/978-3-319-07425-2_3 . hal-01060325

\section{HAL Id: hal-01060325 \\ https://hal.inria.fr/hal-01060325}

Submitted on 3 Sep 2014

HAL is a multi-disciplinary open access archive for the deposit and dissemination of scientific research documents, whether they are published or not. The documents may come from teaching and research institutions in France or abroad, or from public or private research centers.
L'archive ouverte pluridisciplinaire HAL, est destinée au dépôt et à la diffusion de documents scientifiques de niveau recherche, publiés ou non, émanant des établissements d'enseignement et de recherche français ou étrangers, des laboratoires publics ou privés. 


\title{
Anticipation of ETX Metric to manage Mobility in Ad Hoc Wireless Networks
}

\author{
Sabrine Naimi ${ }^{1}$, Anthony Busson ${ }^{2}$, Véronique Vèque ${ }^{1}$, Larbi Ben Hadj Slama ${ }^{3}$, \\ and Ridha Bouallegue ${ }^{3}$ \\ 1 Laboratory of Signals and Systems Université Paris Sud - Supélec - CNRS \\ ${ }^{2}$ University Claude Bernard Lyon 1 - LIP (ENS Lyon - INRIA - CNRS - UCBL) \\ 3 Innov'COM Laboratory Higher School of Communication - Tunisia
}

\begin{abstract}
When a node is moving in a wireless network, the routing metrics associated to its wireless links may reflect link quality degradations and help the routing process to adapt its routes. Unfortunately, an important delay between the metric estimation and its inclusion in the routing process makes this approach inefficient. In this paper, we introduce an algorithm that predicts metric values a few seconds in advance, in order to compensate the delay involved by the link quality measurement and their dissemination by the routing protocol. We consider classical metrics, in particular ETX (Expected Transmission Count) and ETT (Expected Transmission Time), but we combine their computations to our prediction algorithm. Extensive simulations show the route enhancement as the Packet Delivery Ratio $(P D R)$ is close to 1 in presence of mobility.
\end{abstract}

Keywords: ETX, Metric, Ad Hoc, Wireless Network, Mobility, Anticipation

\section{Introduction}

Recent developments in wireless communication technologies and the emergence of mobile units (laptop, smart-phone, etc.) have made possible access to the network anywhere at any time. As the node may be mobile while staying connected, mobility management has to be efficiently performed to ensure a good quality of experience to the users. In cellular networks or IEEE 802.11 infrastructure mode networks, mobile nodes are associated with the best base station or access point according to signal strength. Prediction algorithms [1], based on the quality of the wireless links (signal strength, SINR, etc.), may be used to anticipate the association to the next infrastructure point. When the node is moving, these complex handover mechanisms [2] allow the node to associate transparently from an infrastructure point (Base Station or Access Point) to another. If the mobile node is no more in the same IP sub-network, Layer 3 mechanisms such as Mobile IP [3] are provided to operate IP address changes.

These handover mechanisms and the IP mobility management do not hold in mobile ad hoc networks. Indeed, instead of managing a single link, each node 
senses the wireless links with all nodes within its radio range. Moreover, there is no IP address change to manage. Nodes mobility is then managed by the routing process rather than layer 2 handovers and Mobile IP.

An efficient mobility management can then be performed in two ways: an efficient link sensing algorithm may prevent link failure and invalidates links before they are actually unusable [4], or metrics associated to each link may reflect link degradations due to nodes mobility. An increase of these metrics will involve a path change from the routing process that will switch from degrading links to more stable links. Unfortunately, as link sensing is performed at the network layer, there is always a bias between the real link states and the estimated ones. This bias is two-fold: a time shift of the measured quality exists due to frequency of the probes used to manage the link, and a measurement error is caused by the lack of information from lower layers. Metrics obtained from link states information suffer from these two problems. Moreover, metrics dissemination by the routing protocol can cause an appreciable delay between the time at which the link states are estimated and their inclusion in the route calculations. All these uncertainties lead to high packet loss rate in presence of mobility.

In this paper, we introduce a new method to anticipate the values of routing metrics. Our main idea consists in using a prediction algorithm applied to received signal strengths to estimate future values of the metrics in order to compensate the delay involved by the link quality measurements and their dissemination by the routing protocol. This technique is used in infrastructure based networks, but to our knowledge, has not been proposed to anticipate link metrics in ad hoc networks. Our contributions deal with a new metric calculation method to manage the mobility problem. We consider classical metrics, in particular ETX [5] (Expected Transmission Count) and ETT [6] (Expected Transmission Time), but we combine their computation to our prediction algorithm. We focus on mobile ad hoc networks but it does not prevent its use in other contexts. Simulation results of our approach show that when our algorithm is well configured the Packet Delivery Ratio $(P D R)$ is close to $100 \%$ in presence of high mobility.

The paper is organized as follows. In Section 2, related works on prediction algorithm in infrastructure networks and the main routing metrics used in ad hoc networks are presented. Section 3 highlights the problem statement. Section 4 introduces our prediction method to anticipate the future routing metrics values. In Section 5, we detail the method used to predict the signal strength and its performance. Simulation scenarios and results are described in Section 6. We conclude in Section 7.

\section{Related works}

Prediction algorithm. Mobility prediction approaches can detect the next base station for resource reservation prior to the actual handover. This approach has attracted several research interests [1], [7], [8], [9]. The handoff integration in Layer 2 and Layer 3 has been proposed in [10]. The authors describe a predictive 
handoff approach for Mobile IP to support fast or smooth handoff. They use a real-time mobility estimation to predict a future handoff. In [11], the Received Signal Strength Indicator (RSSI) and the direction of mobile node are also used as input parameters to a fuzzy inference system to predict the handover decision. The paper presents Mobility and Signal Strength-Aware Hand-off Decision (MSSHD) approaches to predict the handover decision. A new machine learning based prediction system is considered in [12]. The authors use information available in cellular networks as Channel State Information (CSI) and handover history, and by embedding Support Vector Machines (SVMs) into an efficient pre-processing structure to resolve prediction. All these mechanisms have been designed for cellular networks, or infrastructure based networks, where a mobile only needs to select the best link (associated to the best infrastructure point). These techniques are thus not suitable in ad hoc networks where all links may be potentially used.

Also, many approaches using mobility prediction algorithm have been proposed in the context of ad hoc networks. A mobility prediction based on neural network and a recording the geographical location of nodes is introduced in [13]. In [14], an adaptive mobility prediction method is proposed. It predicts the future distance of 2 neighboring nodes using automaton. A mobility prediction approach based on a hello protocol is developed in [15]. Each node and its neighborhood predict their own position through an autoregression-based mobility model. All these techniques have been designed for ad hoc networks with the aim to predict future nodes location or the distance between them. But generally, nodes do not know their positions as they are not equipped with geolocation devices, and mobility is often unpredictable (due to directions change for instance). Moreover, the variations of the link qualities are not only linked to the distance between nodes, but also to the environment where the node evolves (city, building, etc.) and complex radio phenomena (fading, shadowing, etc.). Therefore, we think a more appropriate link quality prediction method should be based on signal strength rather than geographical locations.

Metrics. Routing protocols use metrics to determine the shortest-path from a source to a destination. A metric is a numerical value associated to each link. When additive metrics are considered, the shortest-path is the one for which the sum of the metrics of its links is minimal.

Metrics are generally not specifically defined to manage mobility but there are a few exceptions. Some metrics try to reflect the mobility of the nodes, for instance the average number of link breaks [16], or the Link Duration [17] (LD) that corresponds to the time where two nodes are within the transmission range of each other. These metrics aim at capturing the nodes mobility, and favor stable routes. But they are strongly dependent on the mobility patterns, useless when all nodes are mobile, and may be counterproductive when all nodes are fixed. Other metrics, not particularly related to mobility could nevertheless be efficient to manage mobility. These metrics are supposed to reflect link quality and increase when a node recedes from a neighbor. The routing protocol may find better routes and switch from receding to more stable links. Therefore, 
these metrics have two important benefits: to manage mobility efficiently and to favor efficient link according to a performance criteria like bandwidth, loss rates, or delay. A lot of metrics have been proposed in literature [18]. In this paper, we focus on the most popular metrics, ETX [5] and ETT [6], which have been shown among the most efficient [18]. Moreover, we present the LD (Link Duration) metric in detail as it will be used in our simulations.

In order to take into account the link quality, more complex metrics have been defined as ETX [5]. Calculation of ETX uses the forward delivery ratio (df) and the reverse delivery ratio (dr) of the link. df is the probability that a data packet reached the destination successfully. $\mathbf{d r}$ is the probability that a data packet is received successfully. ETX is formally defined as follows:

$$
E T X=\frac{1}{d f * d r}
$$

ETX evaluates the mean number of transmissions and retransmissions needed to send a data packet. Therefore, ETX promotes links with low loss rates. But ETX does not distinguish links with different capacities. To solve this problem, the ETT (Expected Transmission Time) metric [6] was proposed. The ETT calculation is based on ETX but takes into account both data packets length $L$ and transmission rates $B$ :

$$
E T T=E T X * \frac{L}{B}
$$

ETT can be seen as the time expected to transmit a packet of length $L$. It multiplies the number of attempts (the ETX metric) to send the packet by the time to transmit the packet on the link $\left(\frac{L}{B}\right)$.

The Link Duration metric [19] is calculated by measuring the life duration of a link between two nodes. In case of mobility, the authors of [20] have shown that LD can be used as a management tool for mobility. For a given sourcedestination pair, the path with the maximum path duration is selected as the best route. The path duration is the minimum LD of all the links forming the path. The idea is to consider that routes with long path duration tend to be more stable. In our simulations, we shall show that it is very dependent on mobility models and wrong in practice.

\section{Problem Statement}

As we claim, ETX and ETT metrics could be efficient to manage mobility. Indeed, when a node is moving away from a neighbor the quality of the link degrades and the Frame Error Rate increases, as well as ETX and ETT metrics. This link metric becoming high, the routing protocol should find paths with lower metrics (if available) and packet loss should be avoided. But a certain number of phenomena prevent the realization of this ideal scenario.

In Fig. 1, we plotted the Frame Error Rate function for two nodes depending on the Signal on Noise Ratio (SNR). In this simulation, SNR is strictly decreasing 


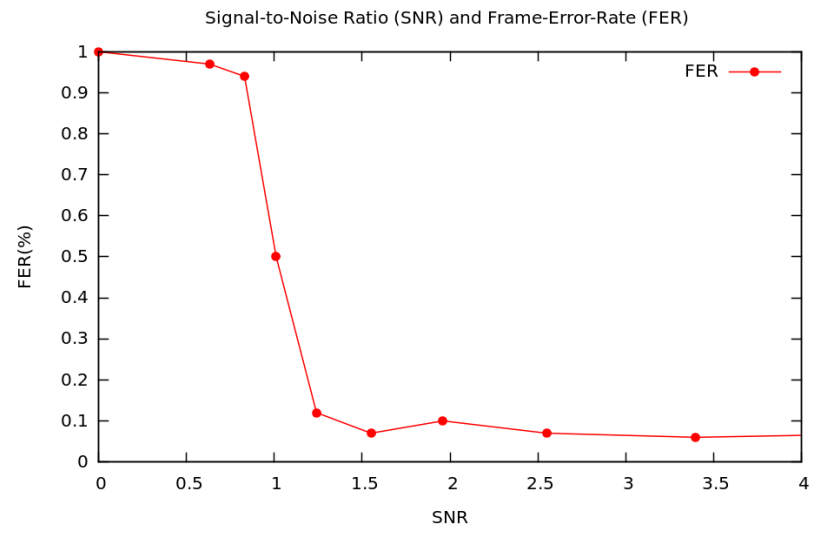

Fig. 1. FER for 2 nodes versus SNR

with the distance according to a path-loss function. It has been obtained with the Network Simulator NS-3 [21]. We observe that FER behaves as a step function. It switches suddenly from $100 \%$ to $5-10 \%$ when the SNR is approximately equal to 1.11 and stays constant otherwise. It means that if a link is receding due to mobility, FER will be close to $5-10 \%$ until a certain distance where it will increase very sharply. This phenomenon is not particular to our model but it is the FER behavior that we observe in general. ETX or ETT will reflect the degradation only when the FER would have reached a high FER. This lossy link will be still used for a while leading to packet losses.

Moreover, calculation of these metrics is generally processed at the network layer and is based on the reception or non-reception of some specific control packets (Hello packets for instance). It introduces a bias in the $d f$ and $d r$ estimations used in ETX or ETT with regard to the instantaneous Frame Error Rate. Also, routing protocols introduce a delay between the ETX/ETT calculations and the time at which they are taken into account in the route computations. These delays are due to the metrics dissemination and the delay to process information and compute routes. All these phenomena add a major delay between the FER degradation and its consideration in the path taken by the data packets. ETX and ETT are then quite inefficient to manage mobility.

The use of information from the physical and MAC layers can help us to estimate instantly and accurately FER, link quality and to prevent link degradation. The main idea of our algorithm is to anticipate the values of link metrics a few seconds in advance. This anticipation allows us to compensate the different delays induced by the FER estimation and the routing protocol. Our mechanism allows these metrics to be also suitable in the context of mobility. Ideally, when our algorithm is perfectly configured, it allows the routing protocol to consider link states in real time and to avoid losses. 


\section{Metric Anticipation Algorithm}

\subsection{Relationship between FER and Signal Strength}

Anticipation is made through a prediction mechanism based on the history of link quality measurements. ETX and ETT metrics are mainly based on the Frame Error Rate of the wireless link. Therefore, it is this quantity that we try to anticipate. But, we do not use previous FER measures since it behaves as a step function. The received signal strength is strictly decreasing/increasing as the nodes are moving and is directly related to FER. It is thus easier to forecast a trend for signal strengths than for FER.

We collect and store the signal strength of the packets exchanged between neighbors for each link. This quantity is generally available through the Received Signal Strength Indicator (RSSI) with 802.11 and 3G/LTE cards. These measures are collected only for control packets as the routing daemon may not be informed of the reception of data packets. We assume that each node has a mapping table to match signal strength to FER. This table can be obtained by the network card manufacturer or inferred through a set of measures performed before the ad hoc network deployment. A function, denoted $F E R($.$) , is then$ used to map a signal strength to a FER. We assume also that FER predictions are exchanged in the Hello packets of the routing protocol. It will allow a node to predict FER for the two directions of a link. The choice of this parameter will be confirmed as shown in our experimentation in Section 5.2.

\subsection{Metric anticipation}

Our proposal consists in using the classical ETX or ETT metrics when the link quality is good, and in increasing artificially and gradually metric values when links start to deteriorate.

The algorithm is presented in Table 1 . It considers the ETX metric, but we could consider ETT as well. The new metric is denoted ETX_ANT. The algorithm is applied at each control packet reception. At each reception, the receiver measures the $L I N K_{-} Q U A L I T Y$, either RSSI, SINR (Signal on Interference plus Noise Ratio), or any quantity reflecting the link quality.

Table 1. ETX_ANT ALGORITHM

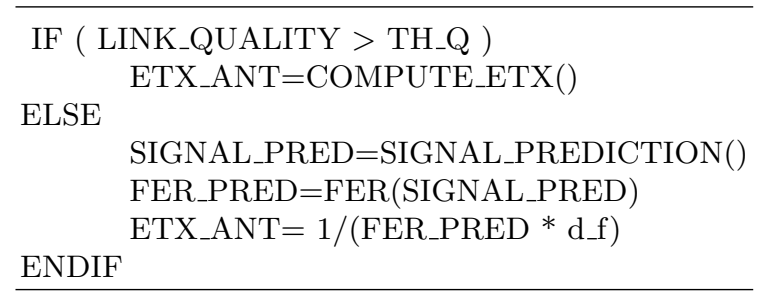


The LINK_QUALITY variable is compared to a predefined threshold denoted $T H_{Q}$. We anticipate the value of ETX, only if the quality is lower than this threshold. First, the signal strength is predicted with the SIGNAL_PREDICTION(.) function using a past measurement history of signal strength values. One possible method to anticipate signal strength is described in the next section. Then, we map this future signal strength to an expected FER with the FER(.) function. It allows the node to estimate the $d_{r}$ parameter of the ETX metric (see Equation (1)). $d_{f}$ is computed on the other end of the link (predicted or not depending on the link quality), and obtained through routing control packets. The ETX_ANT metric is then computed from these anticipated values.

\section{Signal Strength Prediction Algorithm}

Several methods have been proposed to predict signal strength [22]. Most of these methods assume a particular radio environment, through given path-loss or fading. We considered the linear regression method proposed and evaluated in [23] as it has a low complexity and does not rely on a particular radio context.

\subsection{Linear Regression Method}

With a linear regression method, an history of signal measurements, denoted $S=\left\{s_{1}, s_{2}, . ., s_{n}\right\}$, is stored in a table of the last $n$ measurements updated at each new reception. The different times at which they are collected are stored in the vector $T=\left\{t_{1}, t_{2}, \ldots, t_{n}\right\}$. A linear regression model with the form $S=a+b T$ is used to fit the data. The future signal strength is then estimated as:

$$
\hat{s}_{n+p}=a+b p
$$

where $\mathrm{p}$ is the step of the prediction. $a$ and $b$ are given by:

$$
\begin{gathered}
b=\frac{\sum_{i} s_{i} t_{i}-\bar{S} \cdot \bar{T}}{\sum_{i} t^{2}-n \bar{T}^{2}} \\
a=\bar{S}-b \bar{T}
\end{gathered}
$$

$\bar{S}$ (respectively $\bar{T}$ ) is the mean of vector $S$ (respectively $T$ ).

The authors of [23] used a dynamic window (dynamic size of the vectors $S$ and $T$ ) to adapt to abrupt changes. During sudden changes, "old" data would not reflect the current trend, The reduction of the window size is thus necessary to discard measurements that are no more pertinent. The size of the history window is initially set to a default value, and changes according to errors observed in the prediction process. If the prediction error is greater than a threshold, the size of the window will be immediately reduced. However, if the error does not exceed the threshold, the size of the window is increased until it reaches its maximum size. 


\subsection{Experimentations}

We evaluated the prediction algorithm described in the previous Section through a set of experimentations. We configured two laptops in ad hoc mode under Linux (Ubuntu 12.04 LTS). They were equipped with two extern USB wireless cards. We performed this evaluation in the outdoor garden of our laboratory. The two laptops were in line of sight of each other. First, both computers moved in opposite directions at a pedestrian constant speed, then stay more or less at the same distance before approaching a few seconds and moving away for a second time. It aims at evaluating the prediction algorithm when there are clear movements in the signal strength (nodes approaching or moving away) and when the signal oscillates (the distance between the two PCs does not vary much). In Fig. 2,

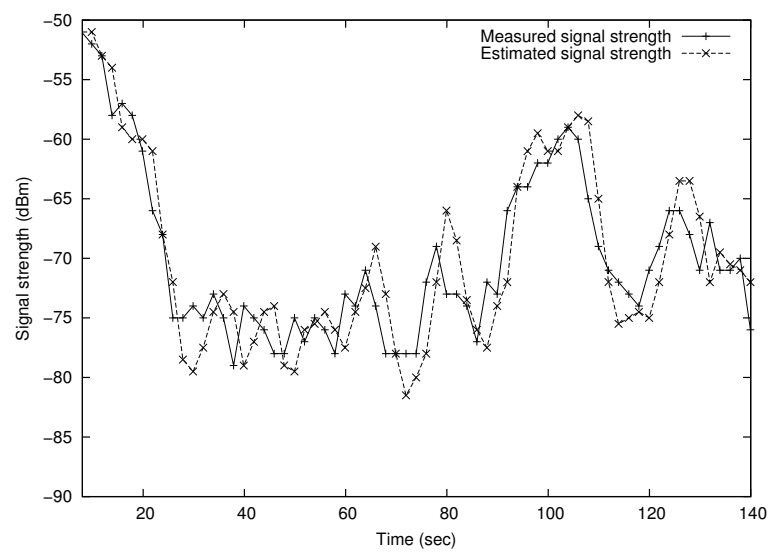

Fig. 2. Comparison of the measured signal strength and the prediction (made 2 seconds in advance).

we plotted the measured signal and the estimated signal strengths $(2$ seconds in advance). It shows that this mechanism successes to predict signal strengths when nodes are approaching and moving away. As expected, when the signal strength oscillates, the prediction is less accurate. Several similar experiments have been performed and led to the same behavior.

\section{Simulations}

Our next evaluation uses simulation in order to consider more complex environment and multihop routes.

\subsection{Parameter Settings}

Before performing the evaluation of our proposal, we need to tune the parameters TIME and $T H_{Q}$. We denote TIME (expressed in seconds) the variable that 
corresponds to the prediction time (the number of seconds we anticipate the signal strength/metric). TIME is related to the variable $p$ in Equation 3. It must be set according to the time needed to disseminate a new metric values in the network and the time to process it (compute routes, etc.). Therefore, it strongly depends on the used routing protocol. In our simulations, we set TIME equal to 2 seconds.

The other parameter, $T H_{Q}$, must be set in such a way that the prediction begins at least TIME seconds before a link breakage. Therefore, a fine tuning of this parameter assumes the knowledge of the radio environment and the maximum relative speed of the mobile nodes. In our scenarios, this information is known. In case of a real deployment, they may be previously inferred through experimentations.

Under the assumption that the physical reception threshold is reached at least TIME seconds after the LINK_QUALITY threshold, we get:

$$
T H_{R e c} \geq P L\left(P L^{-1}\left(T H_{Q}\right)+S_{r e l} \times T I M E\right)
$$

Where $P L$ (distance) is the path-loss function that gives the expected receiving power with regard to the distance, $T H_{R e c}$ is the physical threshold for the frame reception, and $S_{\text {rel }}$ the relative speed between nodes. We get:

$$
T H_{Q} \geq P L\left(P L^{-1}\left(T H_{R e c}\right)-S_{r e l} \times T I M E\right)
$$

\subsection{Simulation Scenarios}

We performed simulations using NS-3. We used the Optimized Link State Routing Protocol (OLSR) [24] because it is a proactive link-state protocol for which it was easy to take into account different routing metrics. Link states information (links and metrics) is disseminated periodically through Topology Control messages (TC). We change the TC message format to include metrics values. Also, we changed the algorithm used to update the routing table. We implemented the Dijkstra algorithm instead of the default algorithm of OLSR, optimized for hop count metric. The rest of the OLSR routing protocol has not been modified. The new metric is disseminated only every TC_INTERVAL seconds, when the node sends its new TC.

We compare our approach (ETX_ANT and ETT_ANT) with the classical metrics ETX, ETT and LD. LD metric is defined by the difference between the last time that the link was symmetric as managed by OLSR and the time at which the TC is sent. The fixed parameters values used for the simulations are given in table 2.

We consider three scenarios: a chain of nodes, a grid network and a completely mobile ad hoc network. Details of these scenarios are described below. In all scenarios, we use the Log Distance Path Loss Model [25] as the radio propagation model. Therefore, we combine this function with a random variable $S$ modeling the channel randomness. $S$ follows a log-normal distribution with zero mean and 
different standard deviations $\sigma\left(\sigma^{2}\right.$ ranges from 0 to 25$)$. The received signal strength is then $P L(d)+S$ (expressed in $\mathrm{dBm})$.

Table 2. Simulation Parameters

\begin{tabular}{|c|c|}
\hline Parameters & Numerical Values \\
\hline Simulation time & $62[\mathrm{sec}]$ \\
\hline Window size (Chain scenario) & $1200 \times 200\left[\mathrm{~m}^{2}\right]$ \\
\hline Window size (Mesh and ad hoc scenario) & $300 \times 300\left[\mathrm{~m}^{2}\right]$ \\
\hline Number of nodes (Chain scenario) & 12 \\
\hline Number of nodes (Grid scenario) & 15 \\
\hline Number of nodes (Ad hoc scenario) & 25 \\
\hline Wireless technology & IEEE $802.11 \mathrm{a}$ \\
\hline Traffic type & Constant Bit Rate (CBR) \\
\hline Packet size & 1024 bytes \\
\hline$T H_{Q}$ & $2.23 \mathrm{mWatt}$ \\
\hline HELLO_INTERVAL (OLSR) & $0.25[\mathrm{sec}]$ \\
\hline TC_INTERVAL (OLSR) & $2[\mathrm{sec}]$ \\
\hline
\end{tabular}

\subsection{Simulation Results}

In all curves, each point is the average of 20 simulations and is shown with a confidence interval of $95 \%$. It may appear that the confidence interval lengths are smaller than the symbols used in the plots, making them barely visible.

\section{Chain Scenario}

The first topology considered is a chain of 11 nodes which are separated from a distance of 100 meters A further mobile node moves at constant speed along the chain of fixed nodes. This topology is a trivial case that allowed us to test the efficiency of our algorithm, and where results are easy to interpret. The source is the mobile node. It transmits data packets at regular interval (CBR traffic). The destination is the first node in the chain. We make varying the speed of the mobile from $10 \mathrm{~km} / \mathrm{h}$ to $70 \mathrm{~km} / \mathrm{h}$. The source moves and the application starts after a few seconds to let the time to OLSR to fill the routing tables. The simulation stops when the mobile node arrives at the end of the chain.

The Packet Delivery Ratio (PDR) is the ratio between the number of packets received by the destination over the number of packets sent by the source. Fig. 3(a) shows the PDR when using different metrics (Hop Count, LD, ETX, ETT, ETX_ANT and ETT_ANT) and varying the speed of the mobile node, and without fading $\left(\sigma^{2}=0\right)$. The PDR with ETX_ANT is $100 \%$ for all speeds. It empirically proves that nodes mobility is perfectly managed 


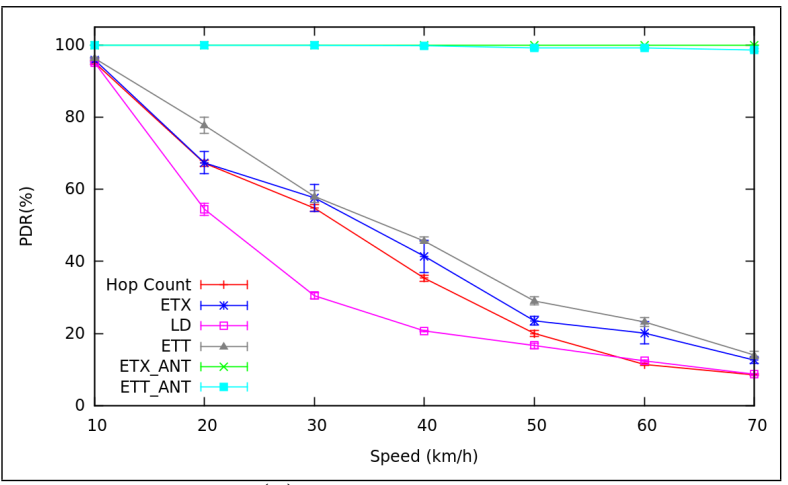

(a) Chain scenario.

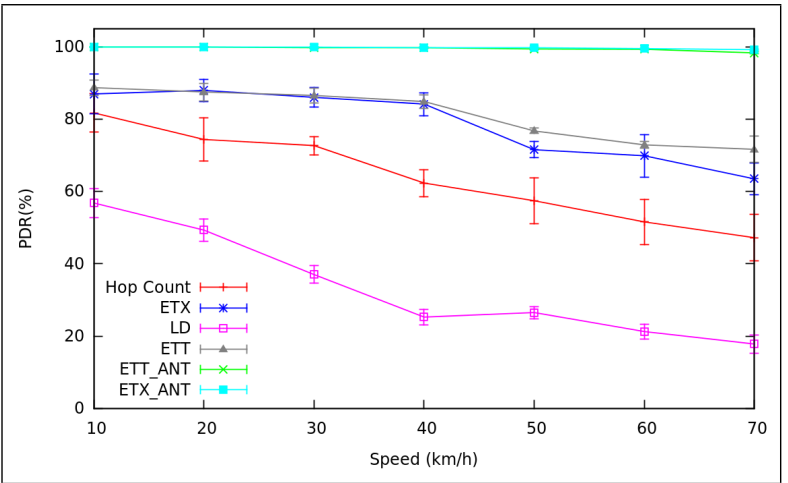

(b) Mesh scenario.

Fig. 3. Packet Delivery Ratio in chain and mesh scenarios.

for this scenario. For higher speed, performance of ETT_ANT decreases, but are greater than $98.5 \%$ and outperforms the classical metrics. The lower performance of ETT_ANT is due to the Wi-Fi rate (parameter $B$ in Equation (2)) that is not predicted in our algorithm, and may lead to inaccurate predictions. LD selects paths with the longest duration which explains why it is the worst metric. Indeed, rather than privileging new links, LD metric chooses the oldest.

\section{Mesh Network Scenario}

In this scenario, we consider a mesh network modeled through a grid topology of 9 fixed nodes. 6 nodes are mobile following the Random Waypoint Model (RWP) [26] with constant speed. We plotted the PDR for the different metrics in Fig. 3(b) when varying the speed. We do not consider fading $\left(\sigma^{2}=0\right)$. Results show that ETX_ANT and ETT_ANT are $100 \%$ for all speeds, proving that our mechanism manages perfectly the mobility in this scenario. 
Ad hoc Network Scenario In the third scenario, we considered an ad hoc network where all nodes are mobile and are randomly distributed in an area with size $300 \times 300 \mathrm{~m}^{2}$. Mobility of nodes follows the RWP model.

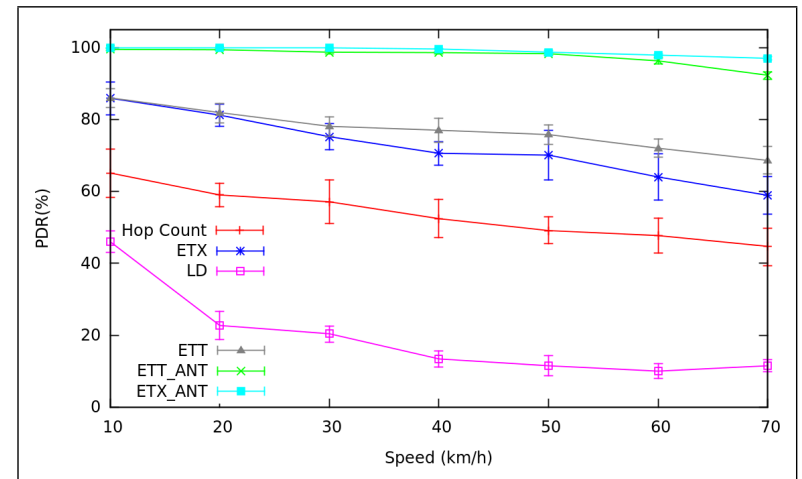

(a) Mobile ad hoc scenario without fading.

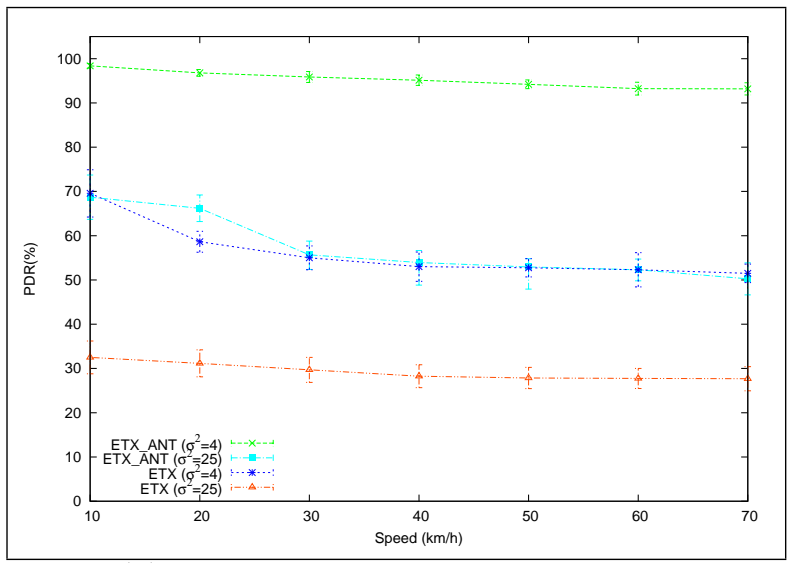

(b) Mobile ad hoc scenario with fading.

Fig. 4. Packet Delivery Ratio in ad hoc scenario with and without fading.

In Fig. 4(a), we can observe that anticipated ETX metric is still very close to 100\%. The PDR with ETX_ANT does not drop below 97\% and ETT_ANT does not exceed $92 \%$. LD metric presents the worst performances: the PDR drops to $11.5 \%$.

To show the impact of fading, we plotted in Fig. 4(b) the metrics ETX and ETX_ANT for different fading value (we vary the standard deviation $\sigma^{2}$ ). We observe that performances degrade when the variance increases whatever the metric. With small fading $\left(\sigma^{2}=4\right)$, PDR with ETX_ANT remains greater than $95 \%$ showing that the mobility is well managed. For $\sigma^{2}=25$, the PDR is at most $70 \%$. Approximately $25 \%$ of the packet losses are due to the fading 
itself and not to mobility (it is the percentage that we observed when nodes were not mobile). This corresponds to a worst case scenario where fading is very important, and where by definition such randomness is unpredictable. But, PDR for the ETX_ANT metric stays more or less constant with the mobile speeds showing that even with high fading it still predicts properly the metrics. By lack of space, these results have not been shown for the previous scenarios, but they present exactly the same behavior.

\section{Conclusion}

In this paper, we have presented an algorithm to anticipate the value of routing metrics based on signal strength prediction. This technique is based on a crosslayer approach. This anticipation allows the routing protocol to compensate the delay due to the computation and dissemination of the metrics. We considered ETX [5] (Expected Transmission Count) and ETT [6] (Expected Transmission Time), but we combine their computation to our prediction algorithm. Through a large set of simulations and scenarios, we have shown that our method improves significantly the PDR. When the network is a Mesh network, where the connectivity is ensured, a proper design of our algorithm leads to a PDR of $100 \%$. For other scenarios, packet losses are provoked by network disconnections and fading, but even in these cases our algorithm outperforms the classical metrics.

This work may be extended in different ways. Particular wireless managers have to be considered in order to combine the FER prediction algorithm and the computation of the future link rate. Also, we plan to deploy our solutions on a testbed to evaluate its efficiency in a real environment.

\section{References}

1. Menezes, S.L.: Optimization of Handovers in Present and Future Mobile Communication Networks. Doctor of philosophy in computer science, University of Texas at Dallas (2010)

2. Shayea, I., Ismail, M., Nordin, R.: Advanced handover techniques in LTE- Advanced system. In: International Conference on Computer and Communication Engineering (ICCCE), pp.74-79 (2012)

3. Perkins, C., Johnson, D., Arkko, J.: Mobility Support in IPv6. Internet Engineering Task Force (IETF), RFC 6275 (Proposed Standard) (2011)

4. Ali, H.M., Naimi, A.M., Busson, A., Vèque, V.:An efficient link management algorithm for high mobility mesh networks. In: 5th ACM international workshop on Mobility management and wireless access (MobiWac), pp. 42-49 (2007)

5. De Couto, D.S.J., Aguayo, D., Bicket, J., Morris, R.: A high-throughput path metric for multi-hop wireless routing. In: 9th annual international conference on Mobile computing and networking (MobiCom), pp. 134-146. New York, USA (2003)

6. Esposito, P.M., al.: Implementing the Expected Transmission Time Metric for OLSR Wireless Mesh Networks. In: 1st IFIP Wireless Days. IEEE (2008)

7. Francois, J.M.: Performing and Making Use of Mobility Prediction. Doctor thesis, University of Lyte (2007) 
8. Yavas, G., Katsaros, D., Ulusoy, Ö., Manolopoulos, Y.: A Data Mining Approach for Location Prediction in Mobile Environments. Data Knowl. Eng. 54, 121-146 (2005)

9. Bergh, A.E., Ventura, N.: Prediction Assisted Fast Handovers for Mobile IPv6. IEEE MILCOM. Washington D.C. (2006)

10. Zhang, Da-Wei., Yao, Yi.: A Predictive Handoff Approach for Mobile IP. In: International Conference on Wireless and Mobile Communications (ICWMC), pp. 78 (2006)

11. Sadiq, A.S., Abu Bakar, K., Ghafoor, K.Z, Gonzalez, A.J.: Mobility and Signal Strength- Aware Handover Decision in Mobile IPv6 based Wireless LAN. In: International MultiConference of Engineers and Computer Scientists, Hong Kong (2011)

12. Chen, X., Mériaux, F., Valentin, S.: Predicting a User's Next Cell With Supervised Learning Based on Channel States. CoRR (2013)

13. Kaaniche, H., Kamoun, F.: Mobility Prediction in Wireless Ad Hoc Networks using Neural Networks. CoRR (2010)

14. Mousavi, S. M., Rabiee, H.R., Moshref, M., Dabirmoghaddam, A.: Model Based Adaptive Mobility Prediction in Mobile Ad-Hoc Networks. International Conference on Wireless Communications, Networking and Mobile Computing (WiCom), pp. 1713-1716 (2007)

15. Li, X., Mitton, N., Simplot-Ryl, D.: Mobility Prediction Based Neighborhood Discovery in Mobile Ad Hoc Networks. Networking 2011, pp. 147-159. Valencia, Espagne (2011)

16. Qin, L., Kunz, T.: Mobility Metrics to Enable Adaptive Routing in MANET. In: IEEE International Conference on Wireless and Mobile Computing, Networking and Communications (WiMob), pp.1-8 (2006)

17. Sadagopan, N., Bai, F., Krishnamachari, B., Helmy, A.: PATHS: analysis of PATH duration statistics and their impact on reactive MANET routing protocols. In: 4th ACM International Symposium on Mobile Ad Hoc Networking and Computing (MobiHoc), pp. 245-256. Maryland, USA (2003)

18. Campista, M.E.M., al.: Routing Metrics and Protocols for Wireless Mesh Networks. IEEE Network. 22, 6-12 (2008)

19. Gerharz, M., de Waal, C., Frank, M., Martini, P.: Link Stability in Mobile Wireless Ad Hoc Networks. In: 27th Annual IEEE Conference on Local Computer Networks (LCN), pp. 0030-. Washington, USA (2002)

20. Bai, F., Sadagopan, N., Helmy, A.: IMPORTANT: a framework to systematically analyze the Impact of Mobility on Performance of Routing Protocols for Adhoc Networks. In: Twenty-Second Annual Joint Conference of the IEEE Computer and Communications (INFOCOM), pp. 825-835. IEEE Societies (2003)

21. Network Simulator 3, http://www.nsnam.org/

22. Duel-Hallen, A., Hu, S., Hallen, H.: Long Range Prediction of Fading Signals: Enabling Adaptive Transmission for Mobile Radio Channels. IEEE Signal Processing Magazine. 17, 62-75 (2000)

23. Long, X., Sikdar, B.:A Real-Time Algorithm for Long Range Signal Strength Prediction in Wireless Networks. In: Wireless Communications and Networking Conference (WCNC), pp. 1120-1125. IEEE (2008)

24. Clausen, T., Jacquet, P.: Optimized Link State Routing Protocol (OLSR). RFC 3626 (Experimental) (2003)

25. Erceg, V., al.: An Empirically Based Path Loss Model for Wireless Channels in Suburban Environments. IEEE Journal on Selected Areas in Communications. 17 (1999)

26. Hyytiä, E., Virtamo, J.: Random waypoint mobility model in cellular networks. Wireless Networks. 13, 177-188 (2007) 